\title{
Bisphosphonates differently affect jaw and long-bone marrow cells
}

\author{
Jenny A.F. Vermeer ${ }^{1}$, Ineke D.C. Jansen ${ }^{1,2}$, Greetje A.P. Renders ${ }^{1}$, \\ Teun J. de Vries ${ }^{1,2}$, Vincent Everts ${ }^{1}$ \\ Departments of ${ }^{1}$ Oral Cell Biology \& Functional Anatomy and ${ }^{2}$ Periodontology, Academic Centre for \\ Dentistry Amsterdam (ACTA), University of Amsterdam and VU University Amsterdam, MOVE \\ Research Institute Amsterdam, Amsterdam, The Netherlands
}

\section{Introduction}

Bisphosphonates (BPs) such as zoledronic acid (ZA) are widely used to treat bone diseases. Due to their ability to inhibit osteoclastic bone resorption, they improve bone quality and reduce fracture risk. BPs can lead to osteonecrosis of the jaw, but it is not clear why in particular jaw bone is affected.

\section{Aim}

To get more insight into the pathogenesis of osteonecrosis of the jaw.

\section{Hypothesis}

BPs have distinct effects on bone-site-specific osteoclasts or precursors.

\section{Methods}

Female C57BL/6J mice (6 per group) were injected i.p. with 0.5 $\mathrm{mg} / \mathrm{kg}$ ZA or saline once a week. At baseline and after 1,3 , and 6 months, jaw and long-bone (Lb) marrow cells were isolated (Fig.3) and osteoclastogenesis was induced in vitro. The number of multinucleated TRACP-positive cells was counted (Fig.4). Bone volume (BV/TV) and the degree of mineralization of bone (DMB) of the humeri and mandibles were assessed with microCT (Fig.1,2). Two-way ANOVA, followed by Bonferroni post-tests (Fig.2) or Mann Whitney $U$ Tests (Fig.3,4) were performed to compare ZA treatment and controls. Means and standard deviations are shown.
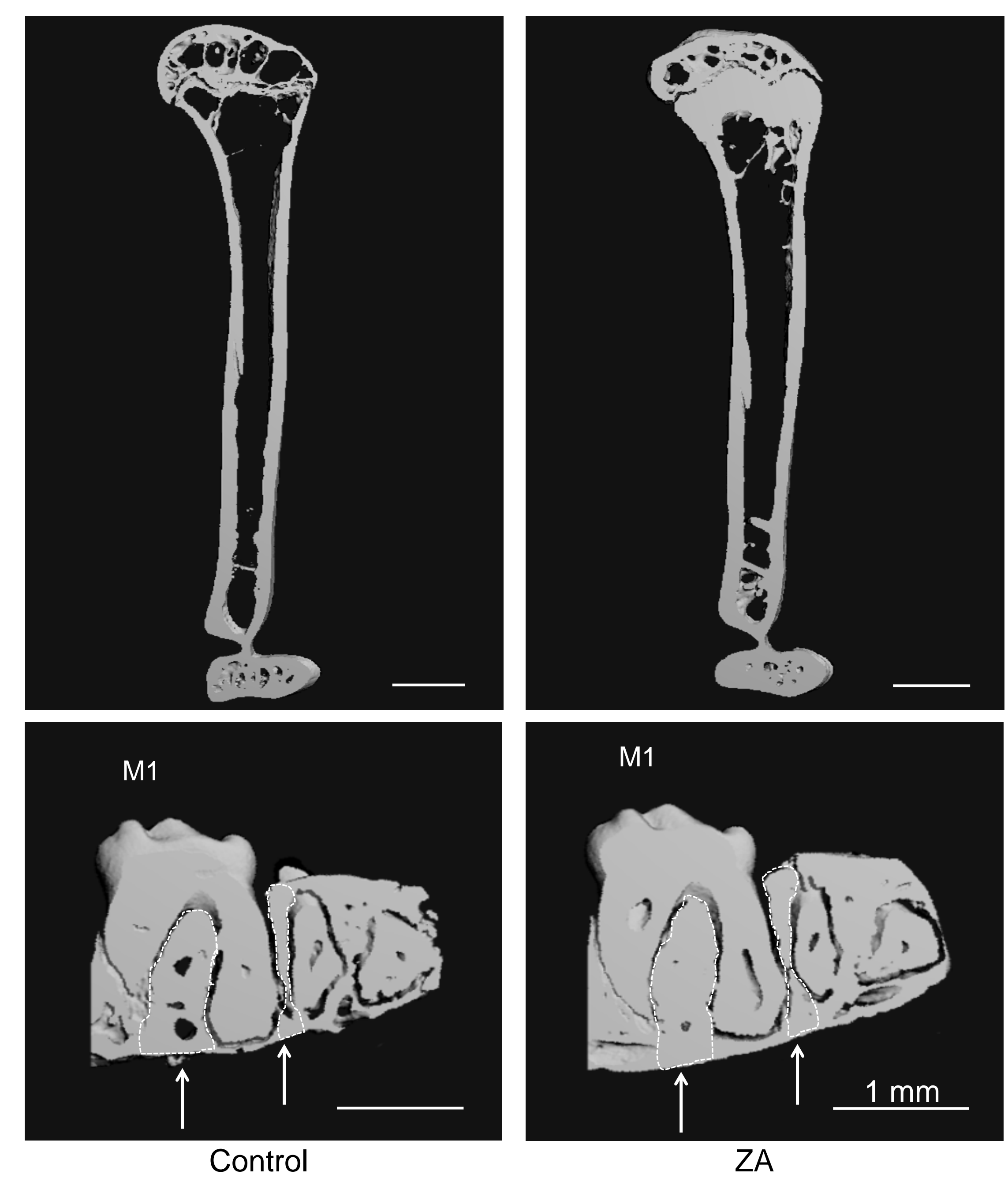

Figure 1. MicroCT images of long bones and jaws, 6 months after starting the treatment. Total long bones (20-30 slices) and for the jaw, areas within the dashed lines (arrows) were analyzed. M1: first molar, bar $=1 \mathrm{~mm}$.

\section{Results}

ZA treatment gradually increased BV/TV and the DMB of both humeri and mandibles.
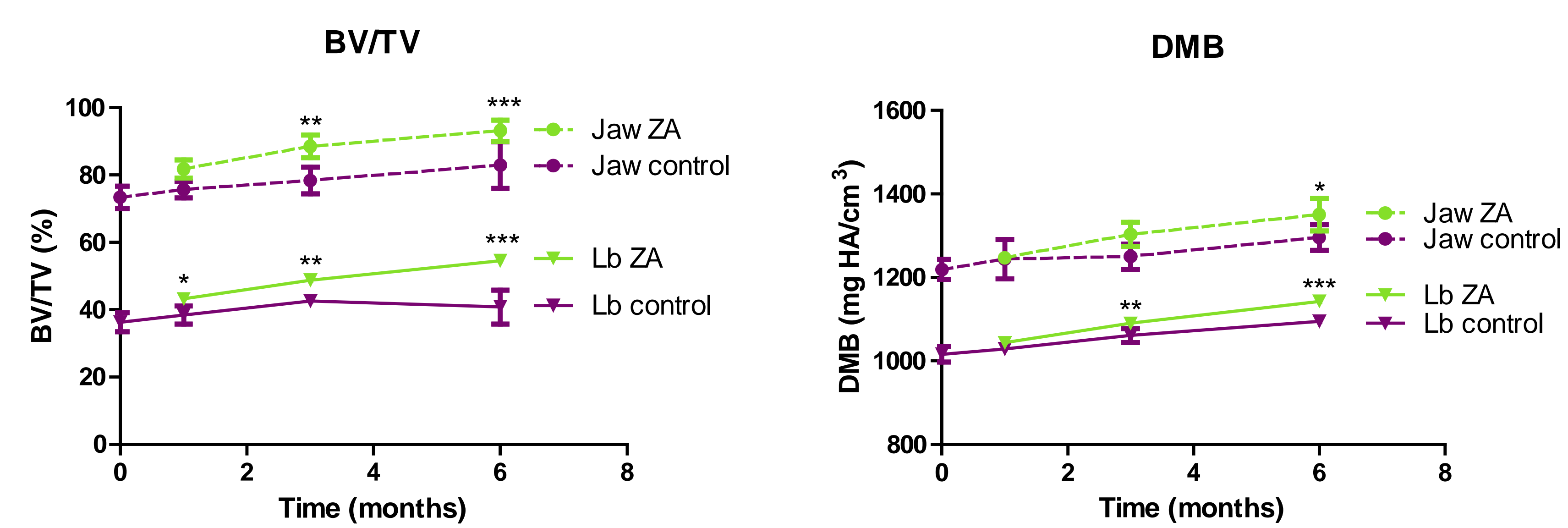

Figure 2. BV/TV (left) and DMB (right) of long bones (Lb, solid lines) and jaws (dashed lines) measured by microCT. ${ }^{*} p<0.05,{ }^{* *} p<0.01,{ }^{* \star *} p<0.001$ represent significant differences between ZA and control.

After 6 months of treatment, fewer jaw bone marrow cells were isolated from ZA-treated mice than from controls. The number of long-bone marrow cells was not affected.
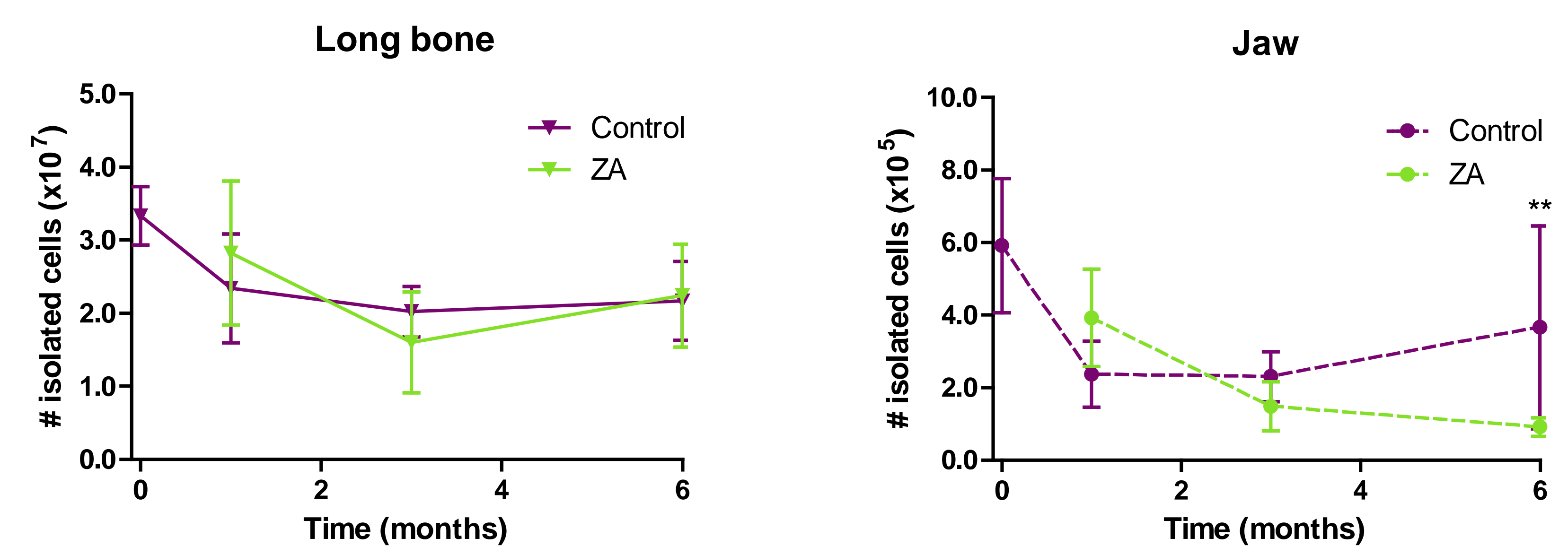

Figure 3. Number of isolated cells from long bones (left) and jaws (right) ${ }^{* *} \mathrm{p}=0.01$ represents a significant difference between $\mathrm{ZA}$ and control.

\section{ZA treatment did not affect the osteoclastogenic potential of long-bone and jaw osteoclast precursors.}
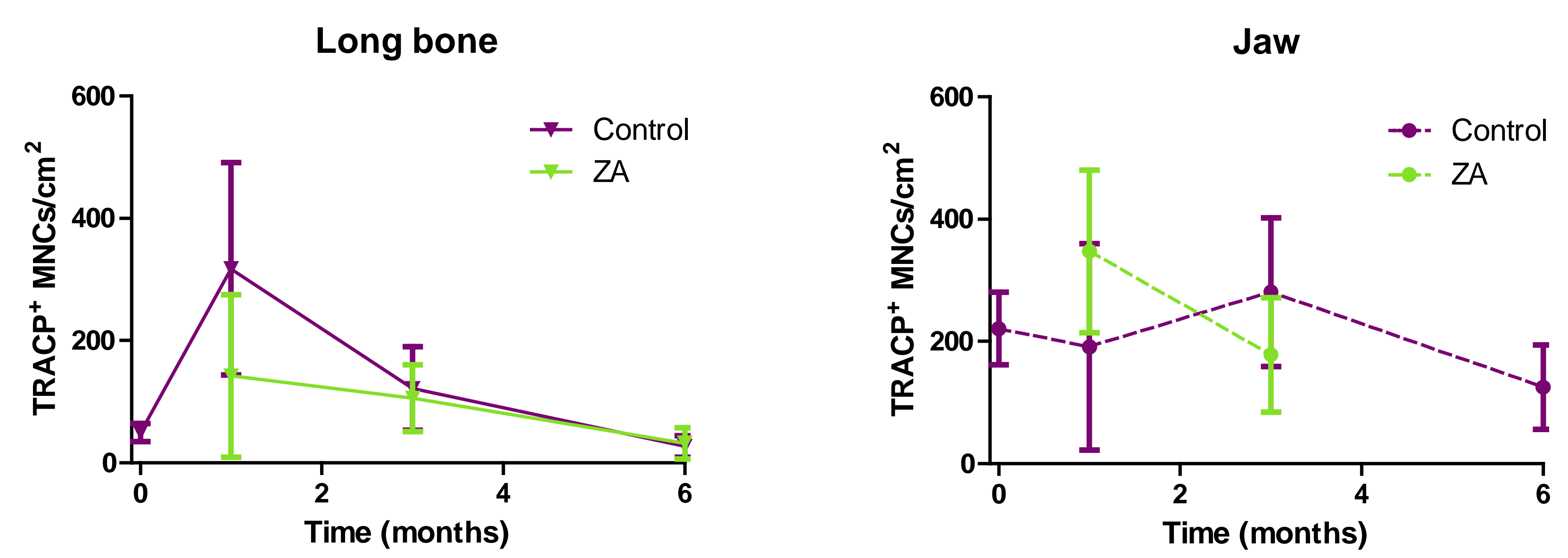

Figure 4. Osteoclast formation from long-bone (left) and jaw (right) bone marrow cells after 6 days.

\section{Discussion}

Six months of ZA treatment significantly reduced the number of bone marrow cells in the jaw without affecting long-bone marrow cells. This is likely the result of a direct toxic effect on jaw bone marrow cells, since bone volume, and therefore bone marrow space was similarly affected in jaw and long bone.

\section{Conclusions}

- Zoledronic acid reduces the number of jaw bone marrow cells without affecting long-bone marrow cells.

- Our findings support the hypothesis that BPs have distinct effects on different osteoclast precursors.

- These results may help to get more insight into the pathogenesis of BP-related osteonecrosis of the jaw. 